\title{
Efficient Dynamic Scheduling Algorithm for Performance evaluation of Workflow in cloud Environment
}

\author{
Prapti S. Deshmukh, Prof. Sneha U. Bohra
}

\begin{abstract}
As of late, distributed computing has been under a creating innovation as a potential answer for giving a flexible, on request figuring foundation for applications. The most testing issue looked by distributed computing is Workflow Scheduling. As, workflow planning is actualized on networks and groups however it shows significant downside, for example, virtual machine execution inconstancy and occasion securing delay. The common framework additionally neglects to either meet the client's Quality of Service (QoS) necessities or to incorporate some essential standards of Cloud registering like flexibility and heterogeneity of the figuring assets. The proposed framework comprises of a dynamic scheduling algorithm for calculation for planning a work process in an open Cloud. This procedure comprises of the preferences offered by the distributed computing for asset the board in IT division by actualizing this calculation to oversee and allot the assets by planning the cut-off times of changed undertakings of significant tasks in IT industry, for example, examination , structure ,usage ,testing, sending and upkeep with less cost .The proposed technique likewise gives the presentation assessment of a representative by breaking down his participation, input from administrator and their partners upheld his work.
\end{abstract}

Keywords - Quality of service, Workflow scheduling, dynamic scheduling algorithm, distributed computing

\section{INTRODUCTION}

Workflows are usually sculptural as group of tasks interconnected via information or computing dependencies. The integration of those tasks onto distributed resources has been studied extensively over the years, specializing in environments like Grids and Clusters. However, with the emergence of recent paradigms like Cloud computing, novel approaches that address the particular challenges and opportunities of these technologies must be developed [2,5]. The Workflow Scheduling has been fully studied but it doesn't show viable solutions. As workflow scheduling is enforced on grids and clusters however it shows major drawbacks like virtual machine performance variability and instance acquisition delay [1]. The National Institute of Standards and Technology (NIST) defines cloud computing characteristics as shown in figure 1

Manuscript revised July 05, 2020 and published on July 10, 2020

Prapti S. Deshmukh, pursuing M.tech (2nd year) from $G H$ Raisoni University, Amravati(Maharashtra)

Prof. Sneha U. Bohra, Head of Department of Computer Science and Engineering in G H Raisoni University, Amravati (Maharashtra).

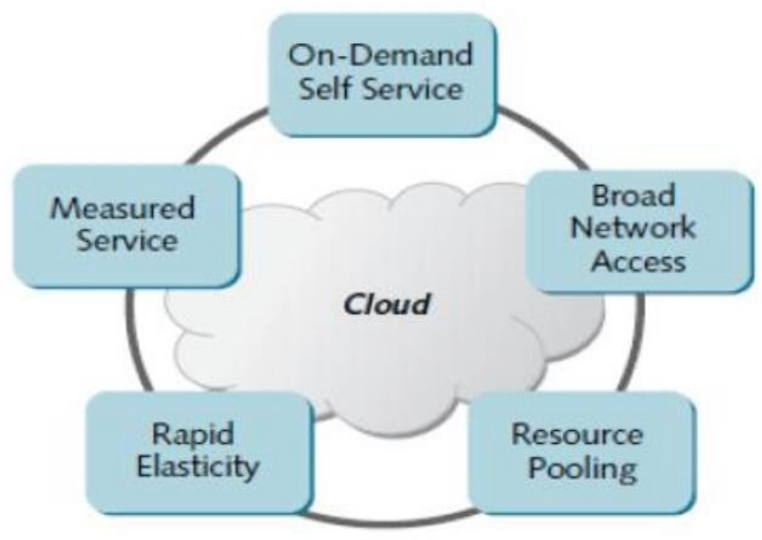

Figure 1: Characteristics of cloud computing

- On-demand self-service: A client will unilaterally provision computing capabilities, like server time and network storage, as needed automatically without requiring human interaction with every service's provider.

- Broad network access: Capabilities are offered over the network and accessed through normal mechanisms that promote use by heterogeneous thin or consumer platforms (e.g., mobile phones, laptops, and PDAs).

- Resource pooling: The provider's computing resources are pooled to serve multiple clients employing a multitenant model, with completely different physical and virtual resources dynamically assigned and reassigned per client demand. There's a means of location independence during this the client typically has no management or information over the precise location of the provided resources however is additionally able to specify location at the subsequent level of abstraction (e.g., country, state, or datacenter).

- Rapid elasticity: Capabilities are going to be rapidly and elastically provisioned, in some cases mechanically, to speedily scale out and rapidly released to quickly scale in. To the patron, the capabilities offered for provisioning usually seem to be unlimited and might be purchased in any amount at any time.

- Measured Service: Cloud systems automatically manage and enhance resource use by investing a metering capability at some level of abstraction applicable to the 
International Journal of Research in Advent Technology, Vol.8, No.6, June 2020

E-ISSN: 2321-9637

Available online at www.ijrat.org

type of service (e.g., storage, processing, bandwidth, and active user accounts). Resource usage are going to be monitored, controlled, and reported providing transparency for each of the provider and client of the used service.

The projected system overcome those drawbacks by implementing a dynamic efficient scheduling algorithm for programming a workflow in a very public cloud. This methodology aims to use the benefits offered by the cloud computing for resource management in IT sector by implementing such dynamic scheduling algorithm to manage and allocate the resources by scheduling the deadlines of varied tasks of major projects in IT industry like analysis, design, implementation, testing, deployment and maintenance and evaluate the performance of an employee.

\section{RELATED WORK}

Mists are being investigated as an answer for some of the issues with Grid processing, yet the contrasts between distributed computing and in this manner the Grid are regularly so decreased and darkened that they become unclear from each other. The expression "Framework" figuring was instituted inside the mid 1990's to compare an appropriated processing foundation to the electric force matrix $[1,2]$.Malawski et.al. proposed different dynamic and static calculations for asset provisioning and planning work process gatherings in Cloud. These calculations expect to amplify the amount of executed work processes while meeting the QoS requirements of cutoff time and spending plan. The proposed arrangements recognize various defers engaged with renting VM assets from the IaaS Cloud, as VM obtaining and end delays. Additionally, the issue of execution variety of VMs is tended to by accepting that an errand's execution time may change bolstered a uniform appropriation. They, be that as it may, consider only one VM type overlooking the heterogeneous idea of IaaS Clouds [3]. Abusayeed, Saifullah, David Ferry, Jing Li, Kunal Agrawal, Chenyang Lu, Christopher Gill learned about Parallel Real time booking of DAG's (directed non-cyclic diagram) which proposed a totally interesting errand disintegration to change over the hubs of an adaptable DAG into successive undertakings. Since every hub of the DAG turns into a private consecutive assignment, these undertakings are regularly booked either preemptively or non-preemptively [4,20]. Saeid Abrishami, Mahmoud Naghibzadeh, and Dick H.J. Epema proposed a substitution QoS based work process booking calculation upheld a totally one of a kind idea called Partial Critical Paths (PCP), that attempts to weaken the value of work process execution while meeting a client characterized deadline [5]. Be that as it may, it gives some time imperative blemishes in execution.Chen and Zhang proposed an Ant Colony Optimization calculation with three QoS boundaries: time, cost and unwavering quality. Szabo at al.proposed a multitarget calculation upheld developmental methodology, for execution of information escalated logical applications during a Cloud situation such the data moved among assignments and in this manner the work process execution time are limited. However, these strategies show great execution they once in a while are longer expending than heuristics approach [6,18].Mao and Humphrey proposed a powerful methodology for planning work process outfits on Clouds such all work processes are done inside their cutoff times at least cost The calculations introduced by Mao and Humphrey were intended for work process troupes and not for single work process examples [7,15].Hoffa, Mehta , and deore additionally considered the work process procedures in distributed computing. The virtual condition can give great process time execution yet it can experience the ill effects of asset booking delays and wide zone correspondences $[8,9]$.Vinay K and S M Dilip Kumar additionally led the analysis on the auto-scaling instrument in distributed computing which is fixed on various Scientific work process and give proficient asset use by considering inert time of Virtual Machines [10,12].Sharif, Taheri and Zomaya et.al proposed Online Multiple Workflow Scheduling under Privacy and Deadline in Hybrid Cloud Environment by presenting OMPHC-PCPR as an additional improvement in online different work process planning calculations. It had assessed the exhibition of the two calculations and delineated that OMPHC-PCPR beats OPHC-TR by lessening the full expense by up to half $[11,19]$.Mattess, Rodrigo N. Calheiros et.al proposed an application situation for the cutoff time mindful execution of MapReduce applications on half breed Clouds. In spite of the fact that this methodology lower multifaceted nature, conveys great outcomes. The network among neighborhood and remote assets are regularly the exclusively predominant factor influencing the culmination time of the full application if the Reduce undertakings don't appear to be computationally concentrated. The proposed calculation defeat of these issues $[13,17]$.Lou and zhang structured a Dynamic Scheduling Strategy for Testing Task in Cloud Computing which limits the makespan and understand the heap balance. Be that as it may, , expanded on cost of cloud asset and along these lines the arrangement space in Genetic Algorithm[14,16].

\section{ISSUES IN WORKFLOW SCHEDULING}

Following are the issues to be satisfied while executing the work process planning for cloud:

- Task-asset planning: Mapping of assets and the booking of assignments of work process in disseminated figuring condition is a NP-Complete problem [2,3]. Such NPComplete issues can be understood utilizing exhaustive hunt procedures. Be that as it may, because of the high multifaceted nature of this methodology ideal arrangement can't be produced inside polynomial time.

- QoS prerequisite: The nature of administration is an imperative issue to live the dependability of the cloud. One of the difficulties presented by cloud applications is Quality-of-Service (QoS) management, that is the downside of distributing assets to the application to guarantee a help level on measurements like execution, openness and responsiveness [5,6].

- On-request asset provisioning: In on-request asset provisioning, an application begins procedure with somewhat number of assets, and it allots the assets when required. Be that as it may, work process planning has a place with NP-hard class of issues, so improvement procedures are favored for the arrangements. 


\section{E-ISSN: 2321-9637}

\section{Available online at www.ijrat.org}

\section{SYSTEM ANALYSIS}

Existing System Issues:

Most of the existing work on workflow scheduling focusses on distributed environments such as Grids and Clusters and very few models are proposed for Cloud based environments $[3,4]$.

\section{Disadvantages of Existing System:}

$>$ A single VM type ignoring the heterogeneous nature of IaaS Clouds.

$>$ Cost is more

$>$ Problem in workflow mapping

\section{Proposed System Design}

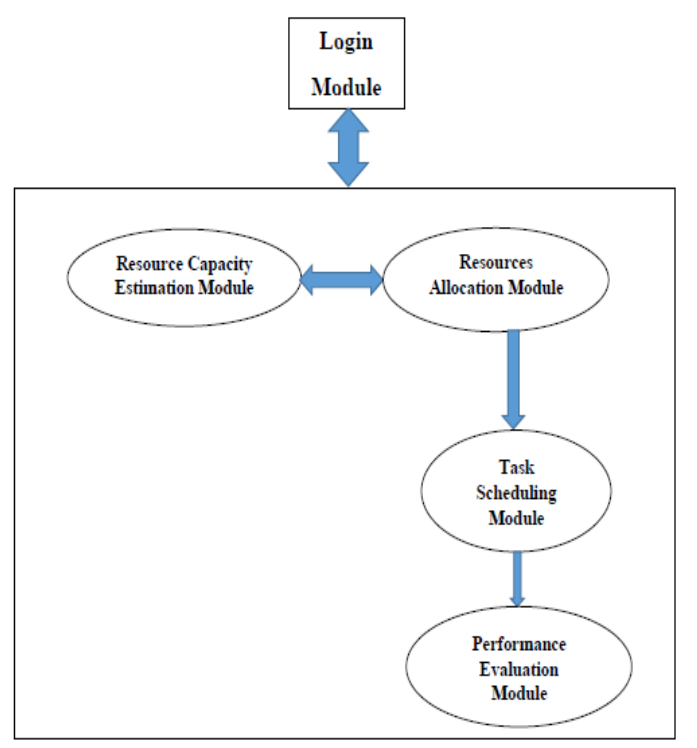

Figure 2: Computing Model for Workflow in Cloud Environment

Above figure 2 shows Computing model for work process in Cloud Environment, the sign in module confirm the qualifications of client and administrator, asset limit estimation module examinations the work process structure to decide the measure of assets required, asset designation module chose the distinguished assets for distributing the undertakings ,task booking module plan the errands of every asset as indicated by their job in IT industry, Performance assessment module investigate the work, participation of a representative dependent on the rating of his/her venture chief and his/her teammates. As appeared in above figure 2 the proposed framework function as follows:

$>$ The provisioning of assets at first recognizes and arrangement the fitting representatives required for the execution of work process of task relegated to IT industry.

$>$ The task execution timetable and need of the errand (i.e high, medium and low) is produced by planning assignments onto proper worker.
$>$ The execution of worker is assessed by his/her evaluations given by the administrator from the participation and his/her errand finishing ability.

Client needs to experience the accompanying strides in figure 3 and figure 4 while managing proposed framework.

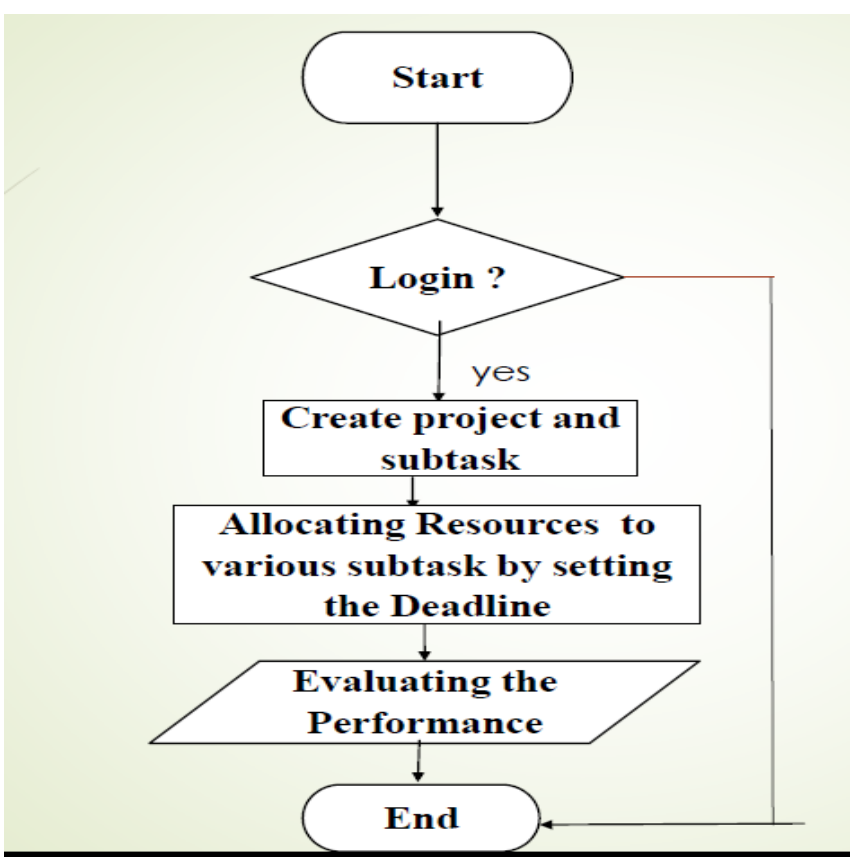

Figure 3: Flowchart of Admin Module

- Firstly, Admin has to login with his id and password, so as to verify the authentication of user over the cloud. By creating the project and schedule the deadline for each subtask, it allocate the resources based on the job profile of an employee.

- $\quad$ Based on the feedback and task completion time gives the rating to the employees.

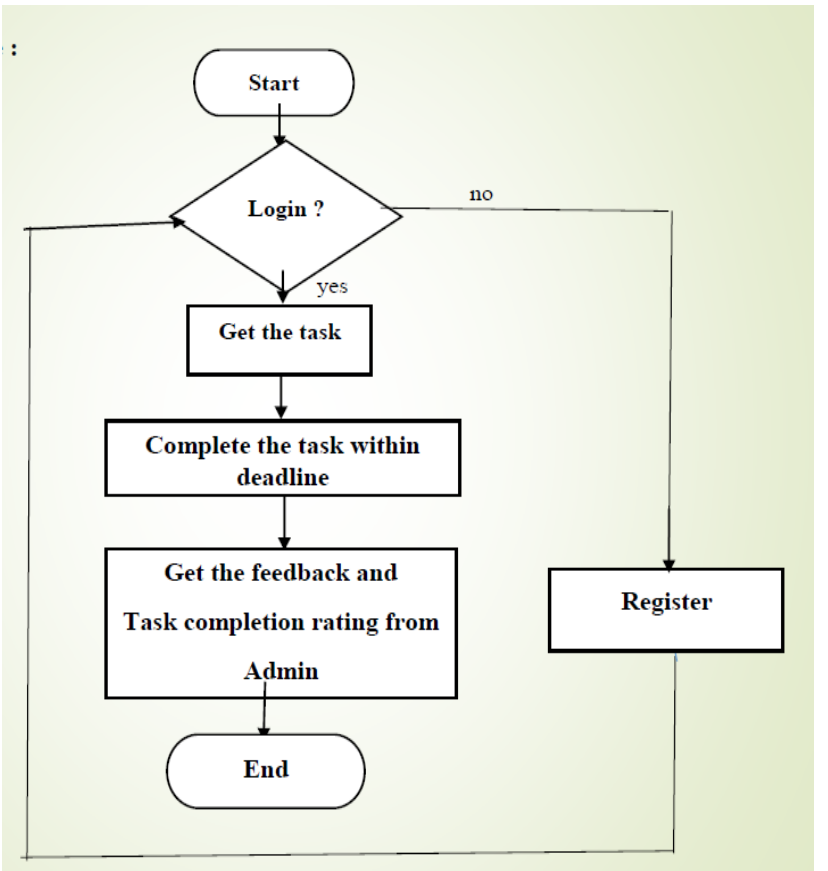

Figure 4: Flowchart of Employee Module 
- In this module, Employee gets his rating and feedback on the basis of task completion based on deadline constraint, which increases his salary and posts.

The proposed technique focuses on provision of managing the resources in corporate sector by using the dynamic scheduling algorithms in cloud environment.It will make cost effective model in order to achieve the true advantages of cloud environment and also evaluate the performance of resources so as to benefit them by provisions.it includes all over essential characteristic features of Cloud and presents dynamic resource provisioning and scheduling algorithm for executing workflows in a Cloud environment that meets the user specified deadline with less cost.

\section{* Performance evaluation Process}

- Assign task to an employee by setting the deadline and priority by admin.

- Analyzing the attendance of an employee by marking the dates where employee login and performs the work and calculates the percentage of attendance.

- Fetches all tasks marks as completed flag by admin and calculate average rating given by admin on each task.

- Calculate the average rating given by the colleague on employee's work.

- Calculate the total task completed and task remain incomplete by employee.

- Generate the graph based on task rating, attendance, and feedback submit by admin and colleagues of the employee.

\section{Proposed System ImPlementation}

\section{- Admin module:}

In admin module, the admin has to login with required credentials and create the project task.After the creation of task by admin ,task is automatically allocated to the required resource by setting the priority and deadline constraint .The workflow scheduling of the project is done by the admin. Project admin evaluates the performance of an resource or employee based on his/her attendance, task completion capability within deadline and feedback of his/her teammates.

\section{- Employee module:}

In employee module, the employee has to login with user-id and password. if employee is newly join to company, he/she has to register with their information and get the user-id and password. The employee has got the task according to his/her designation such as analyst, developer, tester, designer, server manager. After completing the allocated task, employee get rating from admin and feedback about his/her work from teammates.

\section{EXPERIMENT RESULTS AND DISCUSSIONS}

Table 1: Task allocation by comparing the number of previously allocate tasks to which resources are work

\begin{tabular}{|l|l|l|}
\hline $\begin{array}{l}\text { Number } \\
\text { Available } \\
\text { resources }\end{array}$ & $\begin{array}{l}\text { Number of } \\
\text { previous tasks } \\
\text { on which } \\
\text { resource work }\end{array}$ & $\begin{array}{l}\text { Newly assign } \\
\text { task }\end{array}$ \\
\hline R1 & 5 & T4 \\
\hline R2 & 3 & T2 \\
\hline R3 & 4 & T3 \\
\hline R4 & 2 & T1 \\
\hline
\end{tabular}

As shown in table 1, the task allocation is done by comparing the number of tasks which resource has already worked on. For example, here as shown in table 1 , task $\mathrm{t} 1$ is allocated to resource $\mathrm{R} 4$, because it (R4) has less number of tasks to complete.

\section{Conclusion ANd Future SCOPE}

Distributed computing condition offers a great deal of chances and alternatives to execute huge scope enterprises work processes. Executing applications in Cloud includes settles on the suitable provisioning and planning choices so the general execution cost is limited while complying with a client characterized time constraint. To accomplish this, a unique cost-minimization and cutoff time compelled calculation for booking significant assignment during a Cloud situation has been proposed, in order to deal with low execution cost, assets are provisioned not long before they're required. The objective of complying with the time constraint is accomplished through ceaseless observing of the running assignments and it powerfully settles on savvy planning choices for ensuing errands to such an extent that the cutoff time requirement isn't disregarded. At long last, the proposed framework plan to actualize way to deal with keep up a work process of IT enterprises so it is regularly used for expanding the effectiveness and pace of development.

\section{REFERENCES}

[1] Jyoti Sahni and Deo Prakash Vidyarthi(2018) A Cost-Effective Deadline-Constrained Dynamic Scheduling Algorithm for Scientific Workflows in a Cloud Environment, IEEE Trans-actions on Cloud Computing, IEEE Transactions on Cloud Computing, vol : 6, Jan.March 12018.

[2] Rodriguez M. A. \& Buyya R, (2014). Deadline based resource provisioning and scheduling algorithm for scientific workflows on clouds. IEEE Transactions on Cloud Computing, Vol: 2 , Issue: 2 , April-June 12014.

[3] Malawski, M., Juve, G., Deelman, E., \& Nabrzyski, J. (2012, November). Cost-and deadline-constrained provisioning for scientific workflow ensem-bles in iaas clouds. In Proceedings of the International Conference on High Performance Computing, Networking, Storage and Analysis (p. 22). IEEE Computer Society Press.

[4] Saifullah, David Ferry, Jing Li, Kunal Agrawal, Chenyang Lu, Christopher Gill(2013). "Parallel Real-Time Scheduling of DAGs"

[5] Abrishami, Naghibzadeh, Epema(2012 august). Cost-Driven Scheduling of Grid Workflows Using Partial Critical Paths IEEE TRANSACTIONS ON PARALLEL AND DISTRIBUTED SYSTEMS 
[6] Wei-Neng Chen,, and Jun Zhang(JANUARY 2009). An Ant Colony Optimization Approach to a Grid Workflow Scheduling Problem with Various QoS Requirements.

[7] Mao, M., \& Humphrey, M. (2012, June). A performance study on the vm startup time in the cloud. In Cloud Computing (CLOUD), 2012 IEEE 5th International Conference on (pp. 423-430). IEEE.

[8] Deore, and Jaybhaye A Survey on Workflow Scheduling in Cloud Computing IJIRSET.2017

[9] Hoffa ,Mehta, Timothy Freeman, Ewa Deelman2, Kate Keahey3, Bruce Berriman4, John Good . On the Use of Cloud Computing for Scientific Workflows IEEE

[10] Vinay K and S M Dilip Kumarz Auto-scaling for Deadline Constrained ScientificWorkflows in Cloud Environment 2016 IEEE

[11] Sharif, Javid Taheri, Albert Y. Zomaya Online Multiple Workflow Scheduling under Privacy and Deadline in Hybrid Cloud Environment 2014 IEEE 6th International Conference on Cloud Computing Technology and Science.

[12] Chen, Li, Nanjing, Ruben Ruiz "Cloud Workflow Scheduling with Ondemand And Spot Block Instances",Proceedings of the 2017 IEEE 21st International Conference on Computer Supported Cooperative Work in Design.

[13] Matess, Calheiros, and Rajkumar Buyya "Scaling MapReduce Applications across Hybrid Clouds", IEEE 27th International Conference on Advanced Information Networking and Application set Soft Deadlines, 2013 .

[14] Yang Lou, Tao Zhang, Jing Yan, Kun Li, Yechun "Dynamic Scheduling Strategy for Testing Task in Cloud Computing”,2014,Sixth International Conference on Computational Intelligence and Communication Networks.

[15] G. Mohanapriya, Saikishor Jangiti and V.S. Shankar Sriram "Workflow Scheduling in Clouds using Randomized Scheduling Algorithm", International Journal of Pure and Applied Mathematics, Vol:118,2018.

[16] Dr. Amit Agarwal, Saloni Jain "Efficient Optimal Algorithm of Task Scheduling in Cloud Computing Environment" International Journal of Computer Trends and Technology (IJCTT), Vol :9, Mar,2014.

[17] Saeid Abrishami , Mahmoud Naghibzadeh , Dick H.J. Epema "Deadline-constrained workflow scheduling algorithms for Infrastructure as a Service Clouds", www.elsevier.com/locate/fgcs, may 2012.

[18] Anita Choudhary, M.C.Govil, Girdhari Singh, Lalit K. Awasthi "Workflow Scheduling Algorithms in Cloud Environment: a Review, Taxonomy, and Challenges",Fourth International Conference on Parallel, Distributed and Grid Computing (PDGC),2016.

[19] Mainak Adhikari and Tarachand Amgoth "Efficient Algorithm for Workflow Scheduling in Cloud Computing Environment",IEEE 2016.

[20] A. Thushara "Scientific workflow scheduling in cloud computing environment: a survey", International Journal of Computer Engineering \& Technology (IJCET) Vol : 9, Issue 6, NovemberDecember 2018.

\section{AUTHORS PROFILE}

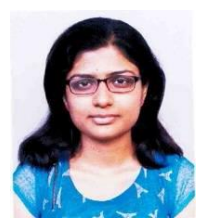

Prapti S. Deshmukh currently pursuing M.tech (2nd year) from $\mathrm{G} h$ raisoni university, Amravati(Maharashtra) and received the B.E degree from Sant Gadge baba Amravati University in 2016.

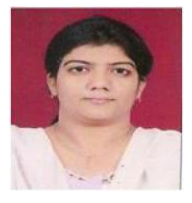

Prof.Sneha U. Bohra is an Head of Department of Computer Science and Engineering in G h Raisoni University, Amravati (Maharashtra). 